\title{
Transthoracic ultrasound-guided percutaneous intramyocardial injection combined with ultrasound-targeted microbubble destruction-mediated angiogenin 1 gene therapy in canine myocardial infarction model
}

\author{
Sheng Cao, Qing Deng, Tuantuan Tan, Yanxiang Zhou, Yijia Wang, Qing Zhou \\ Department of Ultrasound Imaging, Renmin Hospital of Wuhan University, Wuhan, China \\ Contributions: (I) Conception and design: S Cao, Q Deng, T Tan, Q Zhou; (II) Administrative support: S Cao, Q Deng, T Tan, Y Wang, Q Zhou; (III) \\ Provision of study materials or patients: S Cao, Q Deng, T Tan, Y Zhou; (IV) Collection and assembly of data: Y Wang, Y Zhou; (V) Data analysis \\ and interpretation: S Cao, Q Deng, Q Zhou; (VI) Manuscript writing: All authors; (VII) Final approval of manuscript: All authors. \\ Correspondence to: Qing Zhou. Department of Ultrasound Imaging, Renmin Hospital of Wuhan University, 238\# Jiefang Road, Wuchang district, \\ Wuhan, 430060, China. Email: qingzhou.wh.edu@hotmail.com.
}

Background: Intravenous (IV) ultrasound-targeted microbubble destruction (UTMD) has made distinct but limited progress in gene transfected therapy. Intramyocardial (IM) injection also has some effect. In this study, IM injection could be performed under the guidance of transthoracic ultrasound without thoracotomy. We aimed to evaluate the effect of combination transthoracic ultrasound-guided percutaneous IM injection and UTMD for mediating the angiogenin 1 (Ang1) gene therapy in myocardial infarction (MI) canines.

Methods: Forty-two canines were divided into four groups: IM-UTMD group (intramyocardial injection of the negative control plasmid with UTMD as the sham group); IM-Ang1 group (intramyocardial injection of the Ang1 plasmid without UTMD); IM-Ang1-UTMD group (intramyocardial injection of the Ang1 plasmid with UTMD); and IV-Ang1-UTMD group (intravenous injection of the Ang1 plasmid with UTMD). The FITC green fluorescence and Ang1 protein in myocardial tissue were used for the distribution of Ang1 gene. Left ventricular dimension and systolic function were observed by echocardiography. The Masson's and Sirius Red's staining were used for evaluating the collagen fiber. The immunohistochemistry for CD31 and alpha-smooth muscle actin ( $\alpha$-SMA) were as the indicators of microvasculature. Myocardial contrast echocardiography was used to reflect the microvascular perfusion.

Results: More FITC-labeled plasmid was observed in the three IM injection groups than in the IV-Ang1UTMD group, and the Ang1 protein expression was higher in the IM-Ang1-UTMD group. One month later, no significant differences in survival rate and complications were observed among all four groups. Although there were no differences in the left ventricular end-diastolic diameter among the 4 groups, the left ventricular end-systolic diameter was lower in the IM-Ang1-UTMD group than in the other groups. Then, the left ventricular ejection fraction was increased in the IM-Ang1-UTMD group, with lower collagen fiber percentage, higher blood vessel density and myocardial reperfusion intensity than those in the other groups $(\mathrm{P}<0.05)$.

Conclusions: Transthoracic ultrasound-guided percutaneous IM injection combined with UTMD is a safe and effective method for mediating Ang1 plasmid therapy in MI canines.

Keywords: Ultrasound-guided; intramyocardial injection; gene transfection; echocardiogram; myocardial infarction

Submitted Jun 08, 2021. Accepted for publication Sep 29, 2021.

doi: $10.21037 / \mathrm{cdt}-21-364$

View this article at: https://dx.doi.org/10.21037/cdt-21-364 


\section{Introduction}

Cardiovascular disorders are still the primary cause of death worldwide. The intramyocardial (IM) injection of exogenous genes is an effective treatment method that can promote the expression of targeted genes in the myocardium, thereby playing an important role (1). IM injection can be mainly divided into epicardial and endocardial myocardial injection $(2,3)$. Although epicardial myocardial injection-mediated gene treating for ischemic myocardial disease has been shown to be effective, it requires thoracotomy and is very traumatic (4). Endocardial myocardial injection requires the use of special catheters and puncture needles under $\mathrm{X}$-ray and intracardiac echocardiography guidance, which is expensive in terms of materials and difficult in terms of technology. Therefore, both methods are difficult to widely apply.

As a routine and convenient diagnostic tool, ultrasound plays an increasingly important role in clinical interventional monitoring and treatment, and various ultrasound-guided percutaneous puncture treatments are becoming increasingly mature and standardized $(5,6)$. Currently, it is feasible to perform transthoracic ultrasoundguided percutaneous IM injection without thoracotomy. This modality could be used to efficiently and reliably deliver agents or genes to the targeted myocardium region. Maeda et al. (7) described the detailed method of transthoracic ultrasound-guided percutaneous IM injection in a mouse myocardial infarction (MI) model without thoracotomy, including the preparation, material and injection protocol. The China ink, Evans blue or fluorescent microspheres could be used to mark the injection site and to detect successful injection $(8,9)$. Liu et al. (10) even used echocardiography-guided percutaneous IM septal radiofrequency ablation for the treatment of hypertrophic obstructive cardiomyopathy. The advantages of this method include the avoidance of thoracotomy-related morbidities, the ability to select target regions at multiple sites and times, the minimal invasiveness and the low complication rate.

Meanwhile, as a new style of nonviral carrier gene transfection, some studies on ultrasound-targeted microbubble destruction (UTMD)-mediated gene transfection for the treatment of MI. It can promote the angiogenesis of myocardium, alleviate myocardial fibrosis and improve cardiac function $(11,12)$. The mechanism of these effects may be related to the cavitation of UTMD, it can promote the increasing of cell membrane permeability, then allows the exogenous genes to enter the cell and enhance expression of the target protein, resulting in the desired effects (13-15).

Therefore, this study hypothesized that transthoracic ultrasound-guided percutaneous IM injection could successfully transfer the angiogenin 1 (Ang1) gene into the myocardium without thoracotomy and that UTMDmediated gene transfection could further promote myocardial angiogenesis and improve the therapeutic effect on $\mathrm{MI}$ in canines.

We present the following article in accordance with the ARRIVE reporting checklist (available at https://dx.doi. org/10.21037/cdt-21-364).

\section{Methods}

\section{Establishment of MI model by transcatheter coronary embolization}

Forty-two adult male dogs were about $15-20 \mathrm{~kg}$ provided by the Animal Centre of Renmin Hospital of Wuhan University. The experimental protocol was approved by the Ethics Committee of Renmin Hospital of Wuhan University with an ethical license (No.: WDRM20150706), in compliance with the national guidelines for the care and use of Laboratory Animals of the National Institutes of Health. A protocol was prepared before the study without registration. The experimental flow diagram was shown in Figure 1. According to the previous experimental experience of large animals, 6-8 dogs (about 7 on average) were used in each group, and the possibility of sudden death in experimental dogs after myocardial infarction was $25-30 \%$ (according to $30 \%$, survival rate was about $70 \%$ ), the total sample size of the four groups of experiments was at least equal to $40(4 \times 7 / 0.7=40)$. Two of the MI dogs were in poor initial condition, so we made two more dogs in time, using a total of 42 experimental dogs.

The MI model was established by transcatheter coronary embolization, which is consistent with our previous standard procedures $(16,17)$. The anesthesia was induced with an intramuscular injection of ketamine $(100 \mathrm{mg} / \mathrm{kg})$, followed by an intravenous (IV) injection of sodium pentobarbital (30 mg/kg). The dogs were intubated, assisted ventilation, and monitored by electrocardiogram. The FD20 Cath Lab Fluoroscopy Unit (Philips, Andover, MA) and TERUMO cardiac catheter (TERUMO, Tokyo, Japan) were used. The $1,000 \mathrm{IU}$ of heparin were routinely used for intraoperative anticoagulation. A 6.0-Fr. angiographic catheter was used 


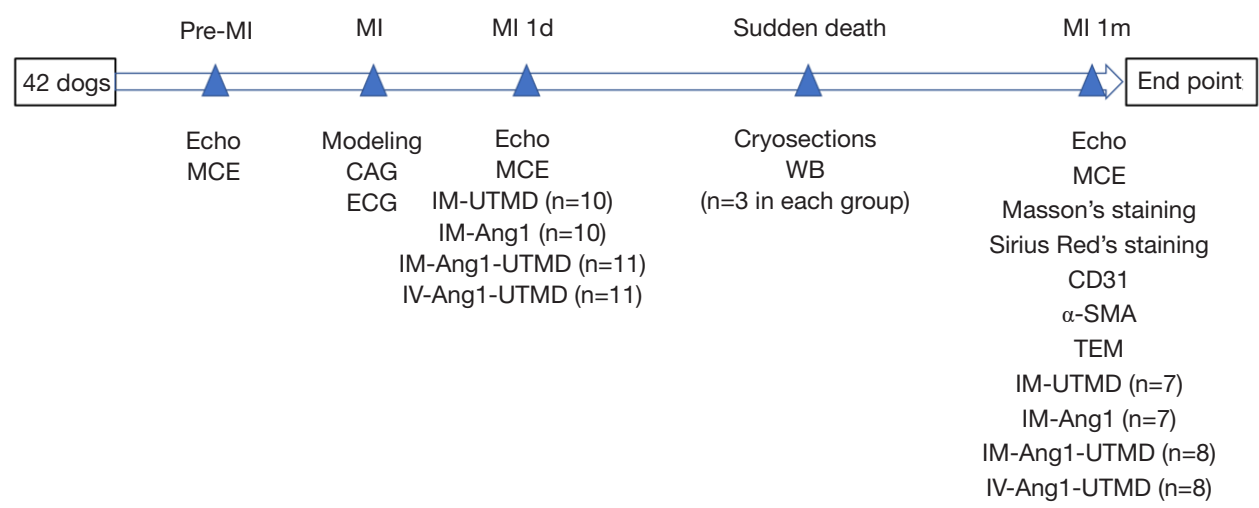

Figure 1 The experimental flow diagram was shown as above. MI, myocardial infarction; Echo, echocardiography; MCE, myocardial contrast echocardiography; CAG, coronary angiogram; ECG, electrocardiogram; IM, intramyocardial; IV, intravenous; UTMD, ultrasoundtargeted microbubble destruction; WB, Western blot; $\alpha$-SMA, alpha-smooth muscle actin; TEM, transmission electron microscopy.

to selectively obtain the left coronary artery angiogram. A 0.014-inch guide wire was inserted for guiding the 1.8-Fr. microcatheter to beyond the second diagonal branch of the left anterior descending artery. The coronary artery was embolized by the injection of 150-350 $\mathrm{mm}$ of gelatin sponge particles (Alicon Pharm Sci \& Tec Co., Ltd., Hangzhou, China) from the microcatheter. $75 \mathrm{mg}$ metoprolol and $50 \mathrm{mg}$ lignocaine boluses were used intravenously to prevent malignant arrhythmia. The MI model was established successfully with significant ST segment elevation, and repeated coronary angiography confirmed myocardial ischemia in the embolized area (Figure 2).

\section{Preparation of FITC-labeled Ang1 carried by microbubbles}

The SonoVue contrast agent (Bracco, Geneva, Switzerland) was prepared by $59.0 \mathrm{mg}$ lyophilized powder and $5.0 \mathrm{~mL}$ of normal saline; following vigorous oscillation, the suspension was formation with approximately $2.0 \times 10^{8}$ microbubbles $/ \mathrm{mL}$. The monolayer of phospholipid consisted of the microbubble envelope containing sulfur hexafluoride with $2.0-5.0 \mu \mathrm{m}$ in size. Then, $1.0 \mathrm{~mL}$ of the Ang1 plasmid (GeneChem, Shanghai, China) solution was added to the $1.0 \mathrm{~mL}$ SonoVue microbubble and incubated for $2 \mathrm{~h}$ at $4{ }^{\circ} \mathrm{C}$. The FITC Tracker Intracellular Nucleic Acid Localization kit (Mirus Bio, LLC, Madison, WI, USA) was used to label the plasmid according to the manufacturer's instructions. The resulting upper layer consisted of microbubbles with attached plasmids; the unattached bottom layer was discarded as previously described (16-18). Finally, the microbubbles concentration was about $1.5 \times 10^{8} / \mathrm{mL}$, with $2.4-7.0 \mu \mathrm{m}$ in size. The concentration of the Ang1 plasmids was approximately $0.25 \mathrm{mg} / \mathrm{mL}$.

\section{Transthoracic ultrasound-guided percutaneous IM injection without thoracotomy}

The transthoracic ultrasound-guided percutaneous IM injection without thoracotomy was performed 24 hours after establishing the MI model. Before transthoracic ultrasound-guided percutaneous IM injection, the skin was disinfected with iodophor, draping with a sterile sheet was performed, and a $20-\mathrm{G}$ puncture needle was sterilized at high temperature for use. The GE Vivid Q ultrasound apparatus (Vista, CA, USA) with a 4C-RS ultrasound probe (frequency, 1.8-6.0 MHz; depth, $7 \mathrm{~cm}$ ) was used to guide the IM injection of $1.0 \mathrm{~mL}$ of the Ang1 plasmid and microbubble suspension through the chest wall into the myocardium. During the injection, the point of the puncture needle and whether the injected suspension was located in the myocardium were clearly visualized by ultrasound display screen (Figure 3). Due to the limitations of the anatomical position and the puncture site, only two sites around the infarcted area were injected at the level of the papillary muscle in the middle of the left ventricle anterior wall, with $0.5 \mathrm{~mL}$ for each position. After the injection, the pericardial effusion was observed, and the changes of ECG were closely monitored.

\section{UTMD for gene transfection}

After the transthoracic ultrasound-guided percutaneous IM injection, the ultrasound irradiation was performed promptly in the targeted region of heart using the GE 

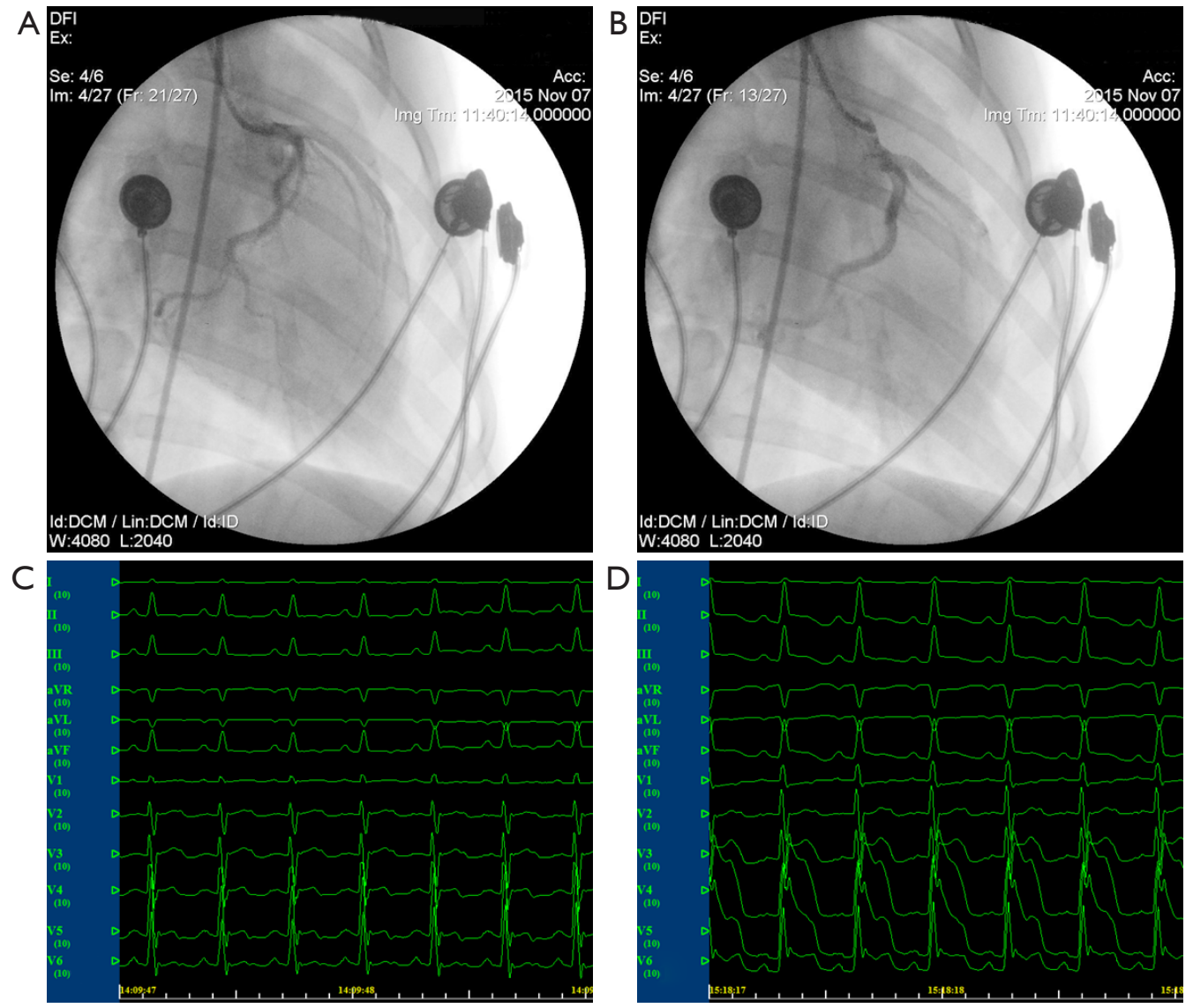

Figure 2 Establishing of the MI model. (A,B) The selective coronary angiogram of left anterior descending artery before and after the MI modeling. (C,D) The electrocardiogram before and after the MI modeling.

Vivid Q ultrasound apparatus with an M4S-RS probe. The ultrasound irradiation parameters were continuous wave, every $10 \mathrm{~s}$ at a $10 \mathrm{~s}$ interval with $50 \%$ duty cycle for $20 \mathrm{~min}$ at a mechanical index of 1.3 , frequency of $1.5-3.6 \mathrm{MHz}$, and depth of $8 \mathrm{~cm}$ in all groups.

The MI model was successfully established in 42 dogs, which were randomly divided into four treatment groups. Random sequence software from the Excel 2016 was used to generate a set of random sequence numbers from 1 to 42. Each dog was assigned a random sequence number, divided by 4 , and then grouped by the remainder. Dogs with the remainder of $0,3,2$ and 1 , respectively entered the IM-UTMD group $(n=10$, transthoracic ultrasoundguided percutaneous IM injection of $1.0 \mathrm{~mL}$ of the negative control plasmid with UTMD as the sham group); the IM-Ang1 group ( $\mathrm{n}=10$, transthoracic ultrasound-guided percutaneous intramyocardial injection of $1.0 \mathrm{~mL}$ of the Ang1 plasmid without UTMD); the IM-Ang1-UTMD group ( $\mathrm{n}=11$, transthoracic ultrasound-guided percutaneous intramyocardial injection of $1.0 \mathrm{~mL}$ of the Ang1 plasmid with UTMD); and the IV-Ang1-UTMD group ( $\mathrm{n}=11$, intravenous injection of $1.0 \mathrm{~mL}$ of the Ang 1 plasmid with UTMD). As above, we used random experimental groupings for dogs. Dr. Cao and Professor Zhou were clearly aware of the experimental procedures, while others were not clear about the specific experimental conditions.

\section{Incidence of complications in MI model dogs}

The incidence of complications of the MI induction procedure and IM injection operation (mortality, true aneurysm, pseudoaneurysm, papillary muscle insufficiency, pericardial effusion, pulmonary edema, pneumothorax and arrhythmia) was recorded from the time of modeling to one month after treatment. The incidence of complications was compared between the groups. 

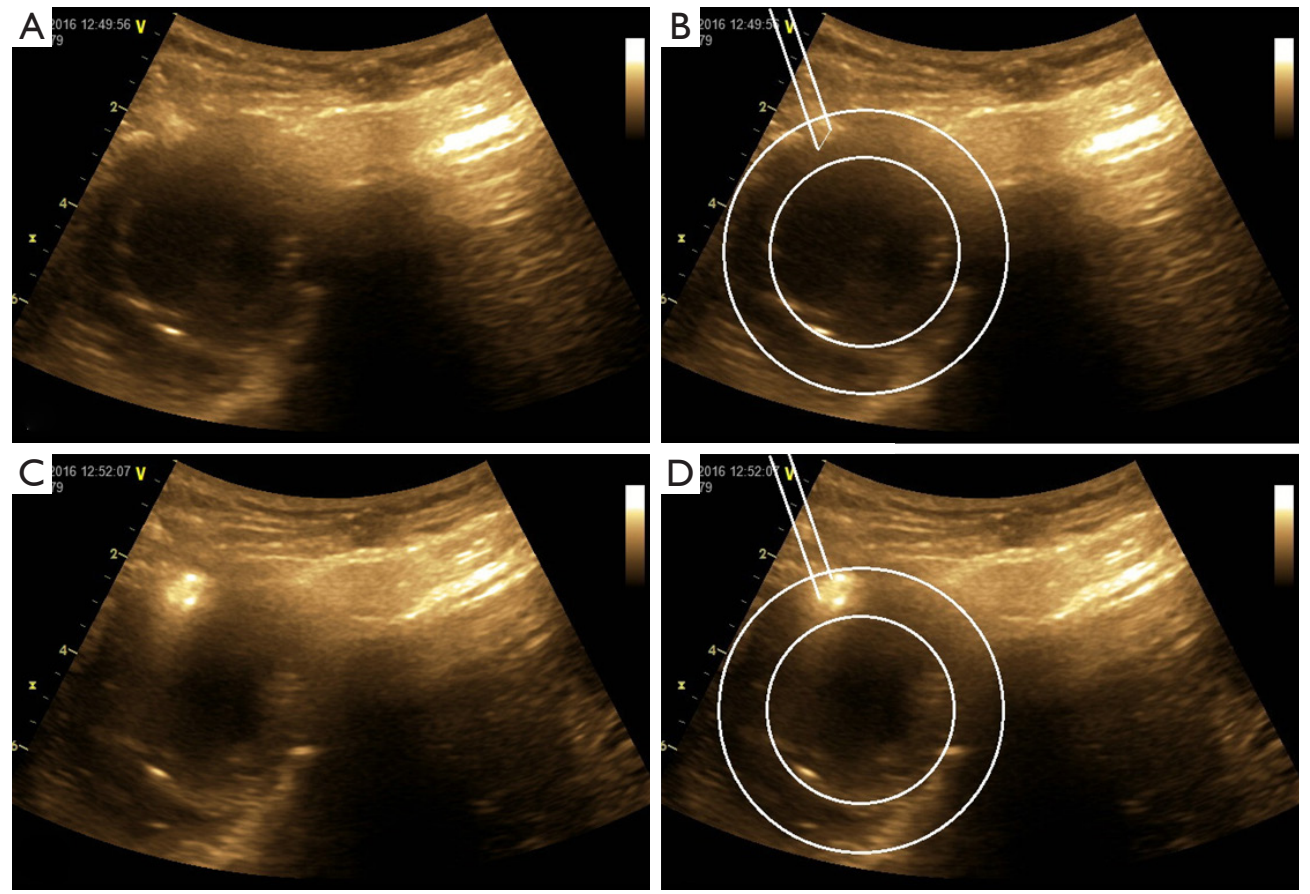

Figure 3 Short axis view of the LV by echocardiography. Schematic diagram of the ultrasound-guided intramyocardial injection process. (A) The needle penetrated the myocardium. (B) Schematic diagram of puncture needle entering myocardium. Between the two circles is the myocardium. (C) The echo of local myocardium was enhanced after the intramyocardial injection. (D) Schematic diagram after the intramyocardial injection; Between the two circles is the myocardium.

\section{Left ventricular (LV) dimension and systolic function observation by echocardiography}

Transthoracic echocardiography was performed in each dog before establishment of the MI model, 1 day after MI and 1 month after treatment. The LV end-diastolic and endsystolic diameter (LVEDD and LVESD, respectively) were measured in 2D mode on the parasternal long-axis view; the LV ejection fraction (LVEF) was calculated using Simpson's biplane method.

\section{Myocardial contrast echocardiography (MCE) for observation of microvascular perfusion}

MCE was performed with the angiographic mode to evaluate myocardial microvascular perfusion before MI, 1 day after MI and 1 month after treatment. After obtaining an adequate acoustic window with suitable depth and gain, the SonoVue contrast agent was infused intravenously. When the contrast agent was filled with plateaued in the myocardium, a flash with high (1.40) mechanical index impulse was given to destroy the contrast agent microbubbles. Then, a low (0.12) mechanical index of MCE was switched on, the myocardial contrast replenishment was visualized and evaluated. The dynamic images were acquired at the parasternal short-axis view of LV midpapillary muscle level for up to 15 cardiac cycles after the flash for offline evaluation. Partial filling defect was observed in the MI area. When perfusion improved, the perfusion defect was reduced.

For qualitative analysis of myocardial perfusion, the region of interest was placed in the middle of each myocardial segment by using Qlab software quantitatively analyzing the reperfusion curve of real-time acoustic radiography. The mean value of three times was taken for the parameters in each region. Plots of time-contrast intensity were constructed and fitted to an exponential function, $y=A\left(1-e^{-\beta t}\right)$. The plateau of signal intensity (A) and the slope of maximum signal intensity rise $(\beta)$ were measured, and the product of $A \times \beta$ was computed. Then the mean value of $A, \beta$, and $A \times \beta$ in each segment was calculated. $A, \beta$, and $A \times \beta$ represent myocardial blood volume, myocardial blood flow velocity, and myocardial blood flow, respectively. The intensity ratio of the contrast agent in the 
infarcted myocardium/noninfarcted myocardium (anterior wall/posterior wall) was calculated to evaluate the relative blood flow volume in the area of MI. The rise intensity of the myocardial perfusion in the infarcted area could be used to reflect the relative blood flow velocity.

\section{Determination of FITC distribution using cryosections}

There were various causes of death after MI. The death of premature dogs was occurred almost within the first week after MI modeling. Because of the experimental animal ethics principle of $3 \mathrm{R}$-reduction, refinement and replacement, they not only could be used to detect the FITC fluorescence distribution under the frozen section to confirm the success of intramyocardial injection, but also extract protein early and indirectly reflect the expression of targeted gene Ang1 after intramyocardial injection. After the sudden death of the MI dogs, the LV myocardium was collected immediately with less than $1.0 \mathrm{~cm}$ from the infarct region and stored at $-80^{\circ} \mathrm{C}$. Cryosections were acquired to assess the distribution percentage of the FITC green fluorescence-labeled plasmids. The mean level of the five visual fields with the highest fluorescence distributions were evaluated in each group. The different fluorescence retention percentage was compared to indirectly reflect the number of plasmids entering the myocardium in all groups (16).

\section{Western blot analysis of Ang1 protein expression}

After the infarcted myocardial tissues were collected from the above sudden death dogs, Ang1 protein was extracted and then separated via $10 \%$ SDS-PAGE according to our previously described standard steps (19). The separated protein was transferred to PVF membranes and incubated with primary antibodies at a 1:1,000 dilution overnight at $4{ }^{\circ} \mathrm{C}$. Both the blocking membrane with $10 \%$ skim milk and the incubating with secondary antibodies at a 1:3,000 dilution needs 1 hour at room temperature. The protein was detected via chemiluminescence and normalized by GAPDH.

\section{Masson and Sirius Red's staining for collagen fiber}

After 1-month, myocardial tissue was collected from all the other dogs. The myocardial tissue at the level of the papillary muscle in the middle of the left ventricle anterior wall was taken to ensure that it was consistent with the ultrasound-guided puncture site. From the general specimen color and texture, it can be seen that the myocardium in the infarcted area was white and harder, which was obviously different from the normal myocardial tissue, which was red and softer. Therefore, we can combine the anatomical location of the ultrasound puncture site and the appearance of the specimen to ensure the accuracy of myocardial tissue extraction. Paraffin-embedded sections of infarcted myocardium were used to evaluate the extent of fibrosis in the infarct area by Masson and Sirius Red's staining. After Masson's staining, normal myocardial tissue appeared red, collagen fibers appeared blue, and nuclei appeared dark blue. After Sirius Red's staining, normal myocardial tissue appeared yellow, collagen fibers appeared red, and nuclei appeared dark red. These differences were used to distinguish normal myocardial tissue from fibrotic tissue. We selected nearly identical sections for both Masson and Sirius Red's staining at the same time for comparison. As described above, the Image-Pro Plus software was used to quantify the five visual fields with the highest collagen fiber percentage.

\section{Immunobistochemistry for CD31 and $\alpha-S M A$ as indicators of microvasculature}

Paraffin-embedded cryosections of the infarcted border zone myocardium were also used to determine the expression levels of CD31 and $\alpha$-SMA and evaluate the density of microvessels. The mean expression levels of these markers were quantified by the same manner as the collagen fiber percentage.

\section{Transmission electron microscopy for ultrastructural observation of myocardial tissue}

During the extraction of infarcted myocardium, a sample of no more than $0.5-1.0 \mathrm{~mm}^{3}$ in size was cut with a new double-edged blade and fixed in $2.5 \%$ glutaraldehyde at $4{ }^{\circ} \mathrm{C}$ for more than $2 \mathrm{~h}$. The sample was then washed with $0.1 \mathrm{M} \mathrm{PB}$ three times for $5 \mathrm{~min}$ each time. After rinsing, it was fixed in $1 \%$ osmium acid at $4{ }^{\circ} \mathrm{C}$ for $2 \mathrm{~h}$ and turned black. After fixation, the sample was washed with $0.1 \mathrm{M}$ PB three times for $5 \mathrm{~min}$ each time. Then, the sample was dehydrated and soaked in a concentration gradient of $50 \%$, $70 \%, 80 \%, 95 \%$, and $100 \%$ anhydrous ethanol (I), $100 \%$ anhydrous ethanol (II), acetone (I), and acetone (II) for 10-15 min each time, followed by acetone and embedding agent $(1: 1)$ at room temperature for $4 \mathrm{~h}$, acetone and 
Table 1 Comparison of incidence of various complications (\%)

\begin{tabular}{|c|c|c|c|c|c|c|}
\hline Complications & IM-UTMD & IM-Ang1 & IM-Ang1-UTMD & IV-Ang1-UTMD & $\chi^{2}$ & $P$ \\
\hline True aneurysm & $2 / 7(28.6)$ & $2 / 7(28.6)$ & $2 / 8(25.0)$ & $3 / 8(37.5)$ & 0.618 & 0.892 \\
\hline Pseudoaneurysm & 0 & 0 & $1 / 8(12.5)$ & 0 & 2.862 & 0.413 \\
\hline Papillary muscle insufficiency & $1 / 7(14.3)$ & 0 & 0 & 0 & 3.348 & 0.341 \\
\hline Pulmonary edema & $3 / 7(42.9)$ & $3 / 7(42.9)$ & $3 / 8(37.5)$ & $2 / 8(25.0)$ & 0.620 & 0.892 \\
\hline Pneumothorax & 0 & $1 / 7(14.3)$ & 0 & 0 & 3.348 & 0.341 \\
\hline Arrhythmia & $3 / 7(42.9)$ & $3 / 7(42.9)$ & $3 / 8(37.5)$ & $4 / 8(50.0)$ & 0.570 & 0.903 \\
\hline
\end{tabular}

IM, intramyocardial; UTMD, ultrasound-targeted microbubble destruction.

embedding agent $(1: 2)$ at $37^{\circ} \mathrm{C}$ overnight, and embedding agent at $37^{\circ} \mathrm{C}$ for $4-5 \mathrm{~h}$. The soaked sample block was placed in an appropriate mold filled with embedding agent, kept at $37^{\circ} \mathrm{C}$ overnight and then moved to $60^{\circ} \mathrm{C}$ the next day. After the samples were stained with lead citrate and uranium acetate solution, ultrastructural changes in organelles could be observed by transmission electron microscopy (HT7700; Hitachi, Tokyo, Japan).

\section{Statistical analysis}

The SPSS 22.0 software (SPSS, Inc., Chicago, IL, USA) was used for statistical analysis. Quantitative data were first analyzed distribution normality by the KolmogorovSmirnov test. The data of normal distribution were compared by ANOVA, and then pairwise comparison was made after the difference was statistically significant. The Kruskal-Wallis $\mathrm{H}$ test was used for the nonnormal distribution data. $\mathrm{P}<0.05$ indicated statistical difference.

\section{Results}

\section{Comparison of survival rate and complications in all 4 groups}

The survival of each group showed that the death of dogs occurred mainly within first week after MI modeling. One month later, 7 dogs survived with $30.0 \%$ mortality in the IM-UTMD group and the IM-Ang 1 group, and 8 dogs survived with $27.3 \%$ mortality in both the IM-Ang1UTMD group and the IV-Ang1-UTMD group. There was no significant difference in mortality among the four groups
(P>0.05, Table 1).

One month after treatment, one dog in the IM-UTMD group showed moderate to severe mitral regurgitation, suggesting papillary muscle insufficiency. One dog with mild pneumothorax happened in the IM-Ang1 group. And only one $\mathrm{dog}$, in the IM-Ang1-UTMD group, developed pericardial hemopericardium after the tip of the puncture needle entered the cardiac cavity during the IM injection process; no increase was observed under continuous monitoring, and a pseudoaneurysm subsequently developed. There were no statistically significant differences in the incidence of complications among the 4 groups $(\mathrm{P}>0.05$, Table 1).

\section{Distribution of FITC green fuorescence in myocardial tissue}

The ratio of FITC green fluorescence was higher in the three IM injection groups (IM-UTMD group, $34.6 \% \pm 3.5 \%$; IM-Ang 1 group, $39.2 \% \pm 5.7 \%$; and IMAng1-UTMD group, $38.5 \% \pm 4.1 \%)$. There were no statistically significant differences among these three groups. The proportion of green fluorescence in the IVAng1-UTMD group was approximately $18.1 \% \pm 3.4 \%$, which was significantly lower than that in the IM injection group $(\mathrm{P}<0.05$, Figure 4). The plasmids were labeled by FITC, then the green fluorescence retention percentage could be used to indirectly reflect the amount of Ang1 plasmid. In other words, the amount of Ang1 plasmid was higher in the IM-Ang1 group and the IM-Ang1-UTMD group than in the IV-Ang1-UTMD group. 

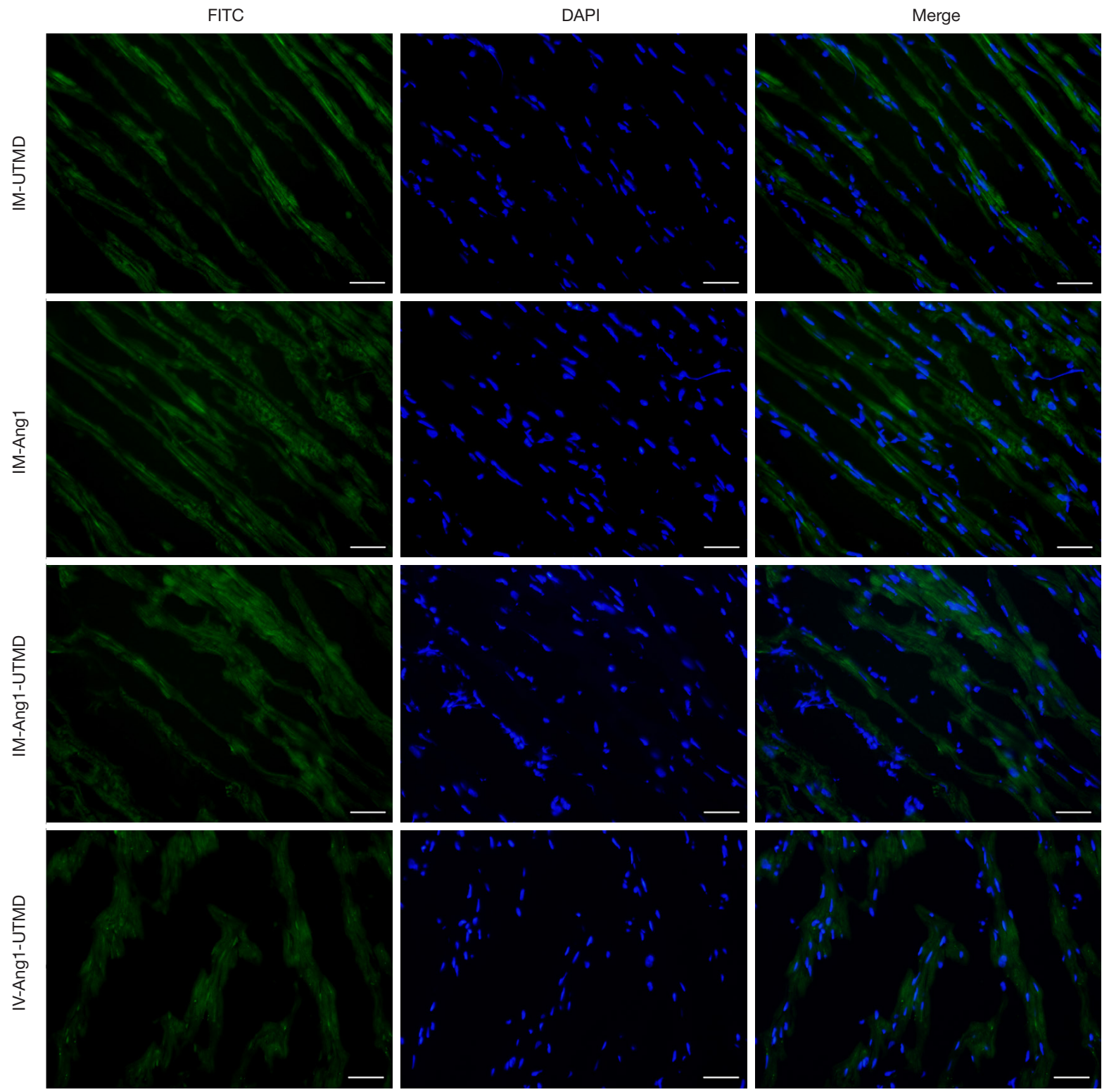

Figure 4 FITC green fluorescence retention percentage was compared in all groups $(\times 400$, scale bar $=50 \mu \mathrm{m})$. The green fluorescence percentage could indicate the relative number of FITC-labelled plasmids, the blue DAPI staining represents nuclei; the merged image consists of green and blue fluorescence.

\section{Western blot analysis of Ang1 protein expression}

The Western blot results showed that the relative expression of Ang1 protein was significantly higher in the IM-Ang1-UTMD group $(54.1 \% \pm 5.7 \%)$ and the IM-Ang1 group $(45.9 \% \pm 5.8 \%)$ than in the IV-Ang1-UTMD group $(34.2 \% \pm 3.9 \%)$ and the IM-UTMD group $(8.5 \% \pm 1.1 \%)$, with significant differences $(\mathrm{P}<0.05$, Figure 5).

\section{Determination of $L V$ dimensions and systolic function by echocardiography}

One day after MI, there were no differences in the LVEDD, LVESD and LVEF. One month after MI, although there were no differences in the LVEDD among the 4 groups, the LVESD was lower in the IM-Ang1-UTMD group $(23.5 \pm 1.9 \mathrm{~mm})$ than in the IM-Ang1 group $(27.5 \pm 2.1 \mathrm{~mm})$ 

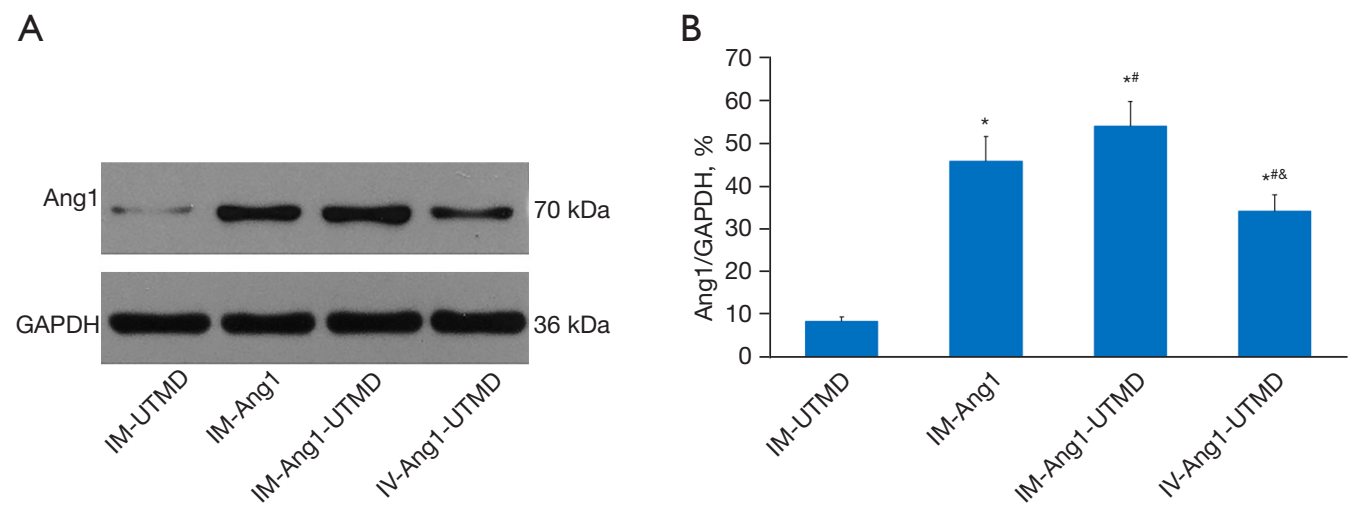

Figure 5 The relative protein expression of Ang1 by Western blot. (A) The Western blot protein bands of Ang1 and GAPDH. (B) Histogram of relative protein expression. The symbol * * and ${ }^{*}$ were represented the comparison with the IM-UTMD group (n=3), IMAng1 group ( $\mathrm{n}=3$ ) and IM-Ang1-UTMD group ( $=3$ ), respectively. Each experiment was repeated three times, with $\mathrm{P}<0.05$.

Table 2 Left ventricular dimension and systolic function detected by echocardiography $\left(\bar{x}_{ \pm s}\right)$

\begin{tabular}{|c|c|c|c|c|}
\hline Parameters & IM-UTMD (n=7) & IM-Ang1 ( $\mathrm{n}=7)$ & IM-Ang1-UTMD (n=8) & IV-Ang1-UTMD $(n=8)$ \\
\hline HR (bpm) & $200 \pm 22$ & $188 \pm 19$ & $183 \pm 21$ & $180 \pm 26$ \\
\hline \multicolumn{5}{|l|}{ LVEDD (mm) } \\
\hline $\mathrm{Ml}-1 \mathrm{~d}$ & $29.5 \pm 2.0$ & $30.7 \pm 1.8$ & $29.9 \pm 0.8$ & $30.1 \pm 1.2$ \\
\hline $\mathrm{Ml}-1 \mathrm{~m}$ & $35.6 \pm 1.9$ & $34.8 \pm 2.7$ & $33.5 \pm 2.0$ & $33.4 \pm 2.5$ \\
\hline $\mathrm{Ml}-1 \mathrm{~d}$ & $22.3 \pm 2.5$ & $23.4 \pm 1.9$ & $22.0 \pm 1.8$ & $23.0 \pm 1.6$ \\
\hline Ml-1m & $30.2 \pm 1.4$ & $27.5 \pm 2.1^{*}$ & $23.5 \pm 1.9^{\text {*\# }}$ & $27.9 \pm 1.8^{* \&}$ \\
\hline \multicolumn{5}{|l|}{ LVEF (\%) } \\
\hline Ml-1d & $40.5 \pm 2.8$ & $39.9 \pm 2.7$ & $41.6 \pm 2.5$ & $39.8 \pm 3.0$ \\
\hline
\end{tabular}

The symbol *, " and \& were represented the comparison with the IM-UTMD group , IM-Ang1 group and IM-Ang1-UTMD group, respectively, with $\mathrm{P}<0.05$. IM, intramyocardial; UTMD, ultrasound-targeted microbubble destruction; HR, heart rate; LVEDD, left ventricular end-diastolic dimension; LVESD, left ventricular end-systolic dimension; LVEF, left ventricular ejection fraction; Ml-1d, 1 day after Ml; Ml-1m, 1 month after MI.

and the IV-Ang1-UTMD group $(27.9 \pm 1.8 \mathrm{~mm})$. The LVEF was higher in the IM-Ang1-UTMD group $(49.4 \% \pm 4.4 \%)$ than in the IM-Ang1 group $(42.6 \% \pm 5.0 \%)$ and the IVAng1-UTMD group $(44.2 \% \pm 3.8 \%)(\mathrm{P}<0.05$, Table 2$)$.

\section{Evaluation of myocardial perfusion by MCE}

The intensity ratio of the infarcted/noninfarcted myocardium (anterior wall intensity/posterior wall) was significantly increased in the IM-Ang1-UTMD group $(0.69 \pm 0.04)$ compared with the other groups (IM-UTMD group, 0.42 \pm 0.04 ; IM-Ang1 group, 0.63 \pm 0.05 ; IV-Ang1-
UTMD group, 0.55 \pm 0.04 ; all $\mathrm{P}<0.05$, Figure 6).

The rate of increase in the intensity of the infarcted myocardium was also higher in the IM-Ang1-UTMD group $(0.081 \pm 0.008)$ than in the other groups (IM-UTMD group, 0.045 \pm 0.006 ; IM-Ang1 group, $0.072 \pm 0.007$; IVAng1-UTMD group, 0.070 0.007 ; all $\mathrm{P}<0.05$, Figure 6).

\section{Collagen fiber percentage as an indicator of myocardial fibrosis}

The results of both Masson and Sirius Red's staining showed the same trend: the collagen fiber percentage was 
A
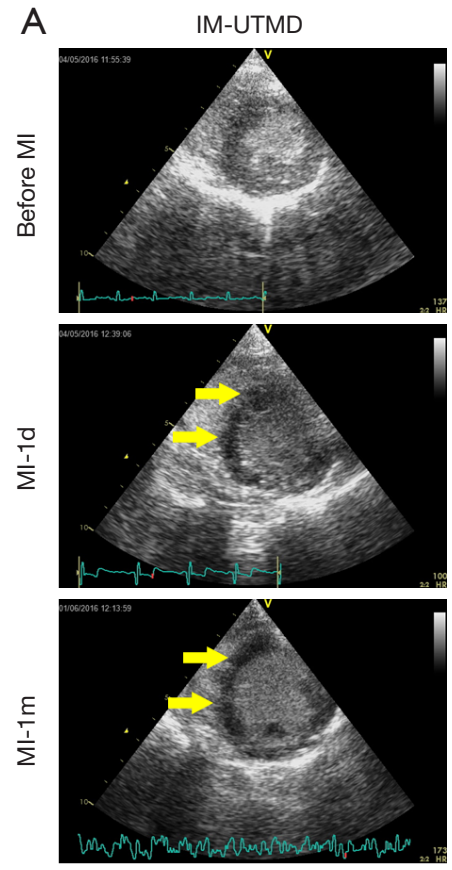

B

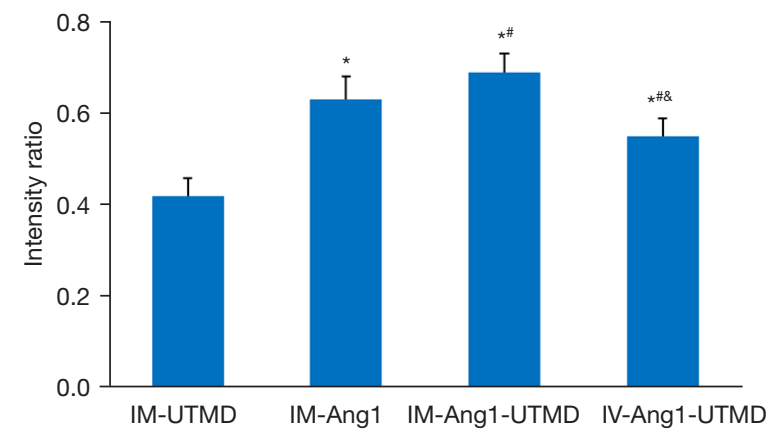

IM-Ang1
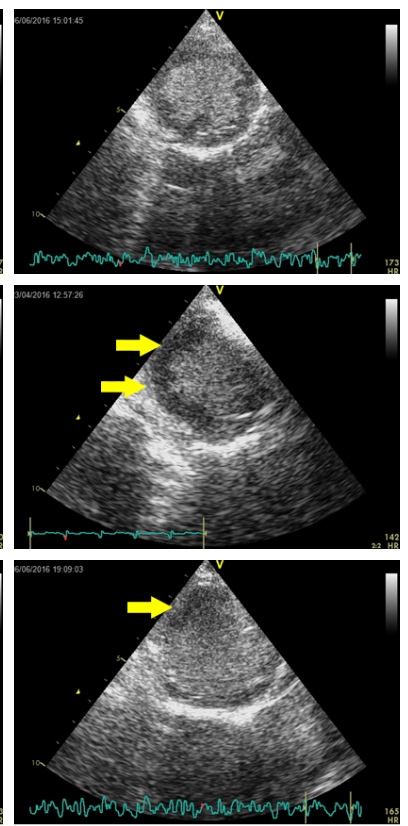

C

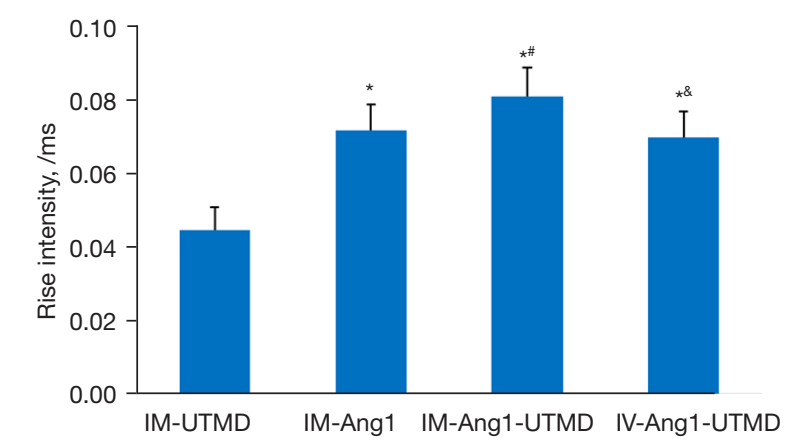

Figure 6 Myocardial contrast echocardiography for microvascular perfusion. (A) Myocardial perfusion was detected before MI modeling, 1 day after MI and 1 month after treatment by myocardial contrast echocardiography. The filling defect was observed in the myocardium area at 1 day after MI. 1 month later, the perfusion improved with reduced perfusion defect area. The yellow arrows represent the filling defect area. (B) One month later, intensity ratio and rise intensity were compared in the 4 groups. The symbol *, ${ }^{*}$ and ${ }^{*}$ were represented the comparison with the IM-UTMD group (n=7), IM-Ang1 group ( $\mathrm{n}=7$ ) and IM-Ang1-UTMD group ( $\mathrm{n}=8$ ), respectively, with $\mathrm{P}<0.05$.

lower in the IM-Ang1-UTMD group than in the other 3 groups (all $\mathrm{P}<0.05$, Figure 7).

\section{Microvessel count by immunobistochemistry}

The results of staining for CD31 expression as a marker of vascular endothelial cells showed that the microvessel density was higher in the IM-Ang1-UTMD group than in the other groups. A similar trend was observed for $\alpha$-SMA expression as a marker of vascular endothelial cells (all $\mathrm{P}<0.05$, Figure 8).

\section{Observation of ultrastructural changes in organelles by transmission electron microscopy}

One month after MI, the myocardial cells in the infarct zone were still slightly swollen, with an irregular arrangement of myofibrils, myofibril breakdown and partial Z-line disintegration. Dense collagenous fiber deposition was observed between cardiomyocytes (Figure 9A).

In the border zone, mild swelling of cardiomyocytes and the basic regular arrangement of myofibrils could be seen. Some mitochondria were swollen, and the extent 
A
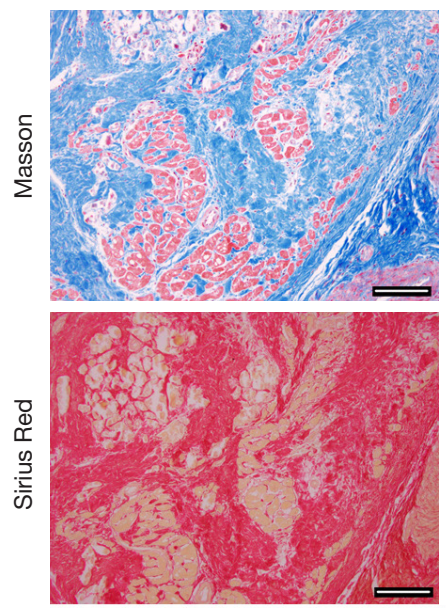

B

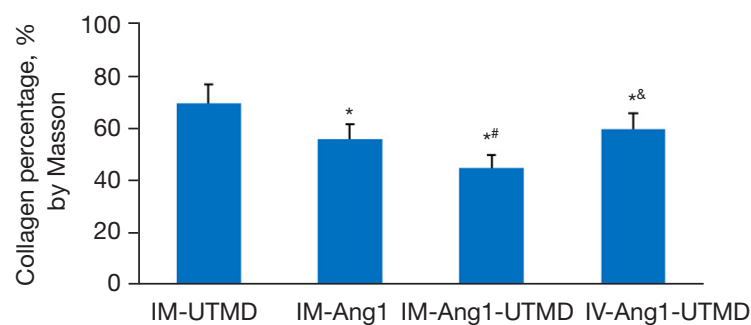

IM-Ang1
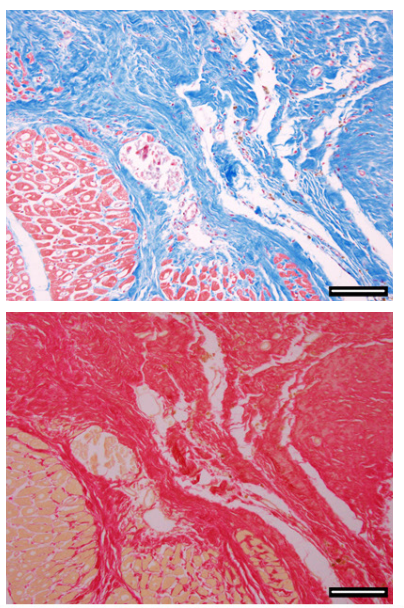

IM-Ang1-UTMD
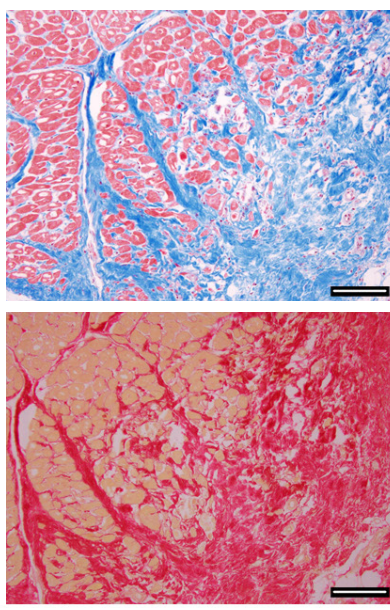

IV-Ang1-UTMD
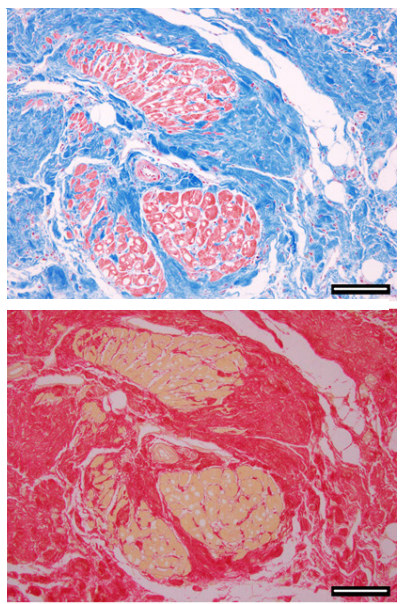

C ஃ

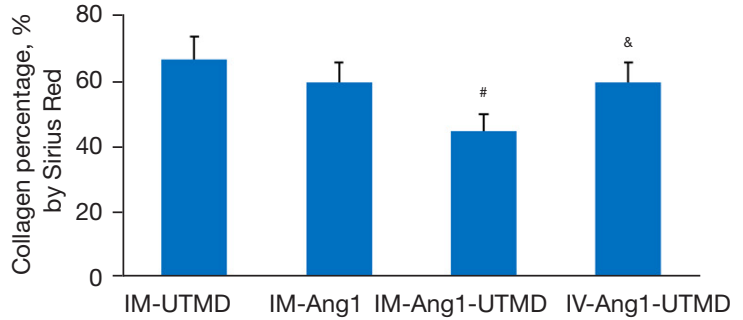

Figure 7 The Masson and Sirius Red's staining of the tissue sections which were obtained 1 month after therapy. (A) In Masson's staining sections, the blue area that indicates collagen fiber and red area that indicates the normal myocardium; in Sirius Red's staining sections, the red zone that indicates collagen and yellow zone that indicates the normal myocardium, respectively $(\times 200$, scale bar $=50 \mu \mathrm{m})$. $(\mathrm{B}, \mathrm{C})$ Comparison of the collagen fiber percentage among the 4 groups based on Masson and Sirius Red's staining. The symbol *, ${ }^{*}$ and ${ }^{*}$ were represented the comparison with the IM-UTMD group (n=7), IM-Ang1 group ( $\mathrm{n}=7$ ) and IM-Ang1-UTMD group ( $\mathrm{n}=8$ ), respectively, with $\mathrm{P}<0.05$.

of myofibril breakdown was slight. Scattered deposits of collagen fibers were observed between cardiomyocytes (Figure 9B).

In the normal zone, regularly arranged myofibrils of cardiomyocytes could be seen, with clear and neat Z-lines and abundant mitochondria. There was no obvious widening of the space between cardiomyocytes, no obvious myofibril breakdown and no deposition of collagen fibers (Figure 9C).

\section{Discussion}

Gene therapy has broad application prospects in the targeted molecular therapy of cardiovascular diseases, especially in the effective delivery of genes to the cardiac region through the selection of appropriate gene carriers and delivery methods (20-23). Currently, commonly used gene vectors include viral and nonviral vectors. Although viral vectors are highly efficient, their safety in vivo cannot be ignored. Nonviral vector transfection methods such as UTMD are still favored by scholars due to their high biosafety despite their relatively low efficiency (23).

At present, most of the experiments involving myocardial injection are performed by direct thoracotomy, especially when coronary artery ligation is used as the modeling method. However, this method has some shortcomings, such as great trauma, difficulty controlling the depth of injection, and inability of the animal experimental environment to completely simulate the general pathophysiological conditions. In large animals, such as dogs and pigs, we can establish the MI model using percutaneous coronary interventional embolization. On the one hand, thoracotomy was not used to establish the canine MI model, and on the other hand, the transthoracic ultrasoundguided percutaneous IM injection did not require thoracotomy. The combination of percutaneous coronary 
A
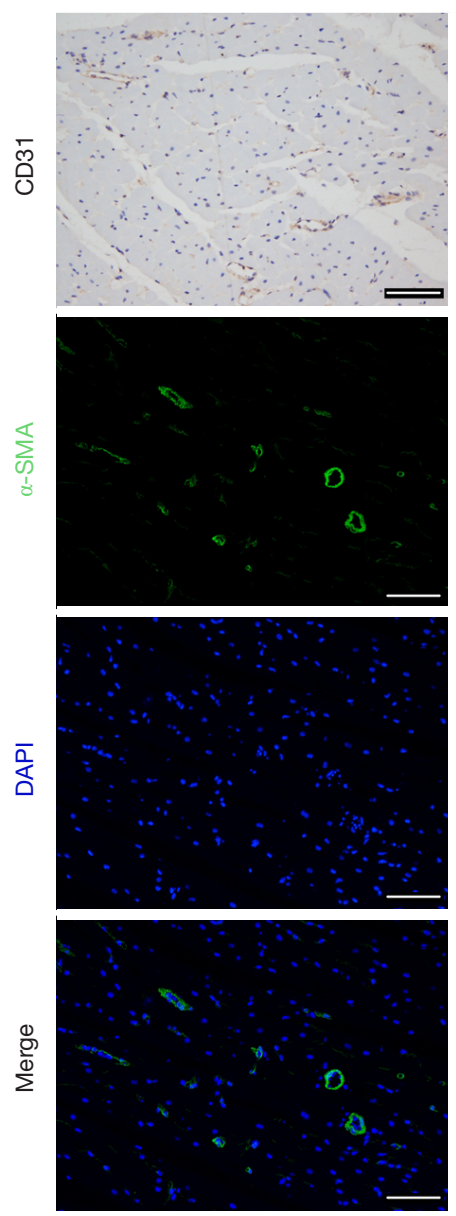

B

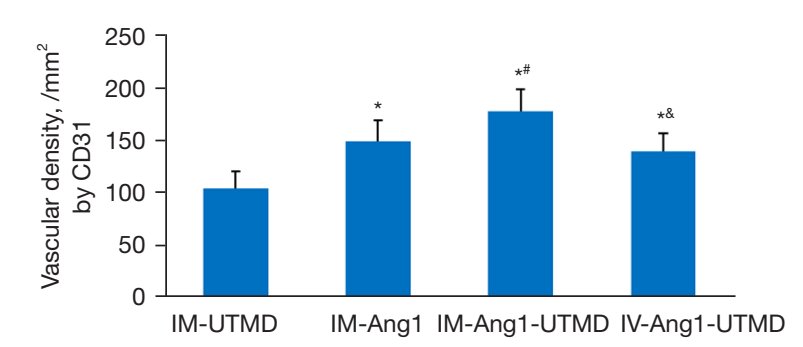

IM-Ang1
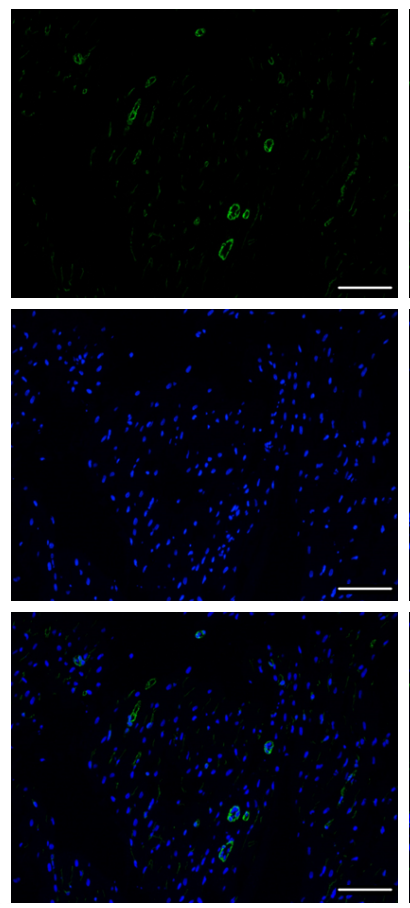

C

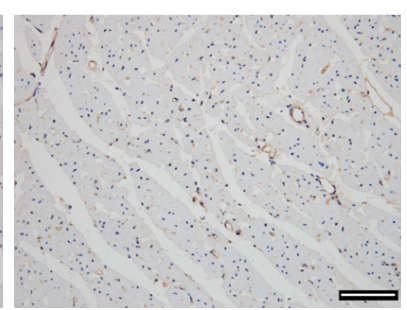

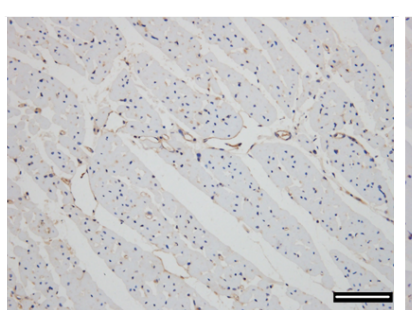
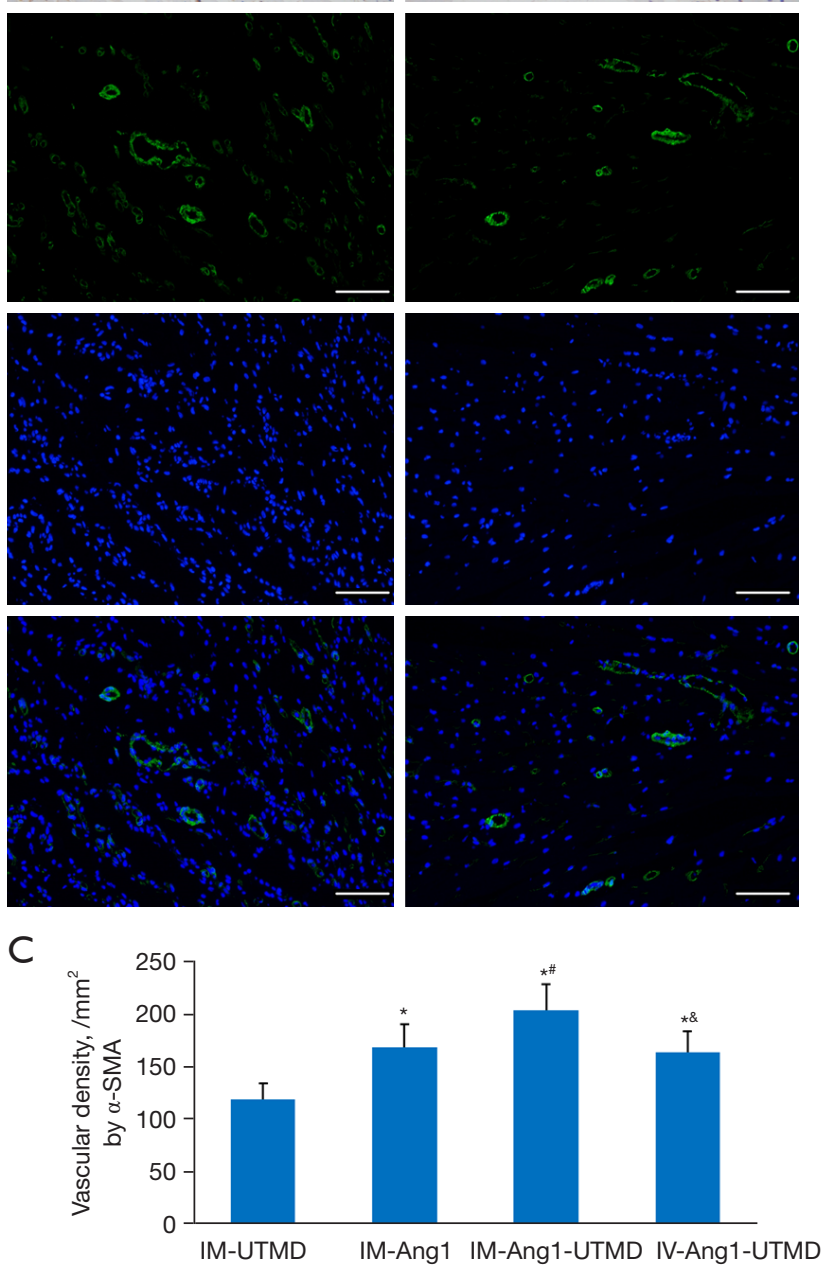

Figure 8 The immunochemistry of the infarcted border zone tissue sections which were obtained 1 month after therapy. (A) The CD31(+) vascular endothelium showed brown and the $\alpha$-SMA (+) vascular endothelium showed green in the sections. The nuclei were stained blue by DAPI, and the merged image consists of $\alpha$-SMA and DAPI staining $(\times 200$, scale bar $=50 \mu \mathrm{m})$. (B,C) Comparison of the microvessel density based on CD31 and $\alpha$-SMA immunochemistry staining. The symbol *, " and * were represented the comparison with the IM-UTMD group ( $\mathrm{n}=7)$, IM-Ang1 group ( $\mathrm{n}=7$ ) and IM-Ang1-UTMD group ( $\mathrm{n}=8)$, respectively, with $\mathrm{P}<0.05$. 

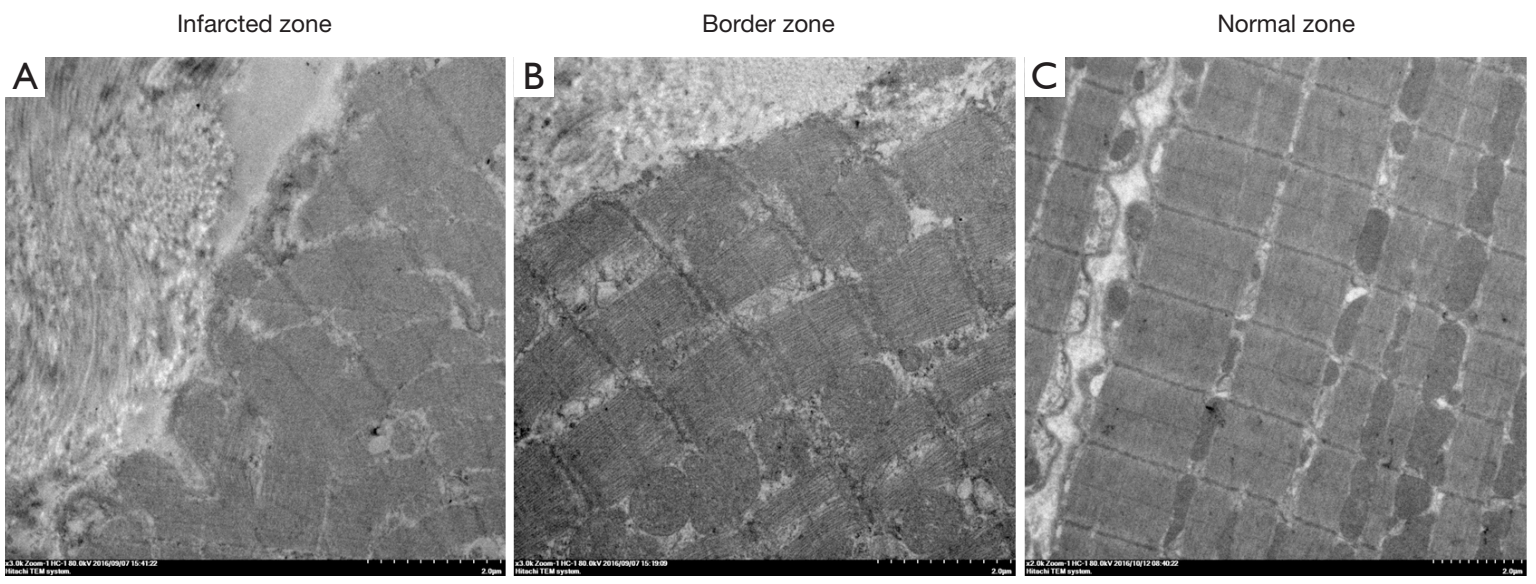

Figure 9 One month after therapy, ultrastructural changes of myocardial organelles by transmission electron microscopy $(\times 3,000$, scale bar $=2 \mu \mathrm{m})$. (A) The myocardial cells in the infarcted zone were slightly swollen, with irregular arrangement of myofibrils, myofibril breakdown and partial Z-line disintegration. Dense collagenous fiber deposition was detected between cardiomyocytes. (B) In the border zone, mild swelling of cardiomyocytes and the basic regular arrangement of myofibrils could be shown. Some mitochondria were swollen and myofibril breakdown was slight. Scattered deposits of collagen fiber were seen between cardiomyocytes. (C) In the normal zone, regular arrangement of myofibrils of cardiomyocytes could be displayed and neat Z-line and abundant mitochondria. There was no obvious myofibril breakdown and no deposition of collagen fiber.

embolization and ultrasound-guided IM injection can not only minimize invasive injury in dogs but also ensure animal safety and better simulate the pathophysiological experimental conditions after MI. Ultrasound-guided realtime monitoring plays an increasingly important role in clinical diagnosis and treatment. In addition to facilitating conventional biopsy, ultrasound can be used in performing some difficult operations step by step, such as ultrasoundguided transthoracic puncture for ventricular septal ablation in hypertrophic obstructive cardiomyopathy (10).

Meanwhile, the ultrasound-targeted transfer of angiogenic gene into the infarcted myocardium is an efficient nonviral vector transfection method. The main mechanism of UTMD is that the cavitation effect can be produced during the burst of microbubbles, which leads to the enhancement of membrane permeability, and exogenous genes can enter cells to enhance target protein expression $(24,25)$. Therefore, in this study, ultrasoundguided transthoracic IM injection combined with UTMDtargeted therapy provided optimized experimental conditions in terms of gene vectors and delivery mode. The results showed no statistically significant differences in the incidence of complications among the four groups, suggesting that transthoracic ultrasound-guided percutaneous IM injection combined with UTMD-targeted therapy is safe and feasible.
Ang1, one of the vascular growth peptides, promotes vascular maturation, proliferation, differentiation, migration and repair, as well as microvascular lumen formation (26). Rufaihah et al. (27) injected vascular endothelial growth factor and Ang1 for MI rats and found that combining them could significantly promote angiogenesis and improve LV systolic function. Our previous studies found that UTMD could effectively mediate Ang1 gene transfection in infarcted myocardium, promote angiogenesis, reduce myocardial fibrosis, and reverse $L V$ remodeling $(17,19)$. The present study shows that the effect of angiogenesis was much better in the IM-Ang1-UTMD group. Moreover, the intensity ratio and rate of increase in the intensity of myocardial perfusion were higher in the IM-UTMDAng1 group, as determined by MCE. These results may be because greater gene expression could be achieved around the infarcted myocardium in the IM injection group than in the IV injection group $(28,29)$ and because sonoporation by UTMD increases the permeability of the cell membrane, promoting gene transfection $(24,30)$. These two factors work together to yield a greater angiogenic effect. Furthermore, the level of myocardial fibrosis was decreased, the LVESD was decreased and systolic function was increased in the IM-Ang1-UTMD group compared with the other groups. These results mean the treatment method can effectively reduce myocardial fibrosis, reverse 
LV remodeling, and improve systolic function in MI.

\section{Limitations}

First, the MI model was established by transcatheter coronary embolization with the gelatin sponge particles, which could be absorbed in about a week. Therefore, the MI model did not achieve early coronary reperfusion in time, revascularization could occur in about a week after the absorbing. That means the time of myocardial ischemia was long and the period of vascular recanalization was relatively late. Second, the positions of the heart and thoracic cavity were relatively fixed, and the chest of the experimental dogs was convex. Because of the irregular thoracic anatomy, there was a risk of complications in transthoracic ultrasound-guided percutaneous IM injection, such as hemopericardium, pneumothorax, and hemothorax. Observation from the right chest showed that the right heart was in front of the image. The right chest myocardial puncture had a greater risk of complications, and the left chest puncture was relatively safer. When in the supine position, the heart moved significantly back resulting in a deeper position. On the one hand, it affected the ultrasound observation. On the other hand, it was not suitable for intramyocardial injection through the subcostal region. So, we can only choose two LV anterior wall injection sites through left chest with ultrasound positioning at the papillary level of parasternal short-axis view. Third, there was injury to local tissue and cells after the myocardial injection, leading to the accumulation of inflammatory factors and inducing the immune response, which may eliminate plasmids to some degree. One study reported that even an injection of more than $0.2 \mathrm{ml}$ into the myocardium could lead to inevitable myocardial injury (31). The total volume of the injection was $1.0 \mathrm{~mL}$ in this study. Four, the heart did not stop when the heart was injected under thoracic ultrasound guidance, which may cause some of the injected suspension containing the Ang1 gene to be lost along with myocardial contraction.

\section{Conclusions}

The transthoracic ultrasound-guided percutaneous IM injection of Ang1 combined with UTMD is a safe and effective gene therapy strategy without thoracotomy, and its mediation of Ang1 expression can effectively promote myocardial angiogenesis, reduce fibrosis, reverse LV remodeling, and improve systolic function in MI.

\section{Acknowledgments}

Funding: This study was supported by the National Natural Science Foundation of China (81901757, 81901759 and $81971624)$ and the Nature Science Foundation of Hubei Province (No. ZRM S2019000321).

\section{Footnote}

Reporting Checklist: The authors have completed the ARRIVE reporting checklist. Available at https://dx.doi. org/10.21037/cdt-21-364

Data Sharing Statement: The authors have completed the Data Sharing Statement form. Available at https://dx.doi. org/10.21037/cdt-21-364

Peer Review File: Available at https://dx.doi.org/10.21037/ cdt-21-364

Conflicts of Interest: All authors have completed the ICMJE uniform disclosure form (available at https://dx.doi. org/10.21037/cdt-21-364). The authors have no conflicts of interest to declare.

Ethical Statement: The authors are accountable for all aspects of the work in ensuring that questions related to the accuracy or integrity of any part of the work are appropriately investigated and resolved. All animals were provided by the Animal Centre of Wuhan University. Experiments were performed under a project license (NO.: WDRM20150706) granted by the Ethical Committee of Renmin Hospital of Wuhan University, in compliance with the national guidelines for the care and use of Laboratory Animals of the National Institutes of Health. A protocol was prepared before the study without registration.

Open Access Statement: This is an Open Access article distributed in accordance with the Creative Commons Attribution-NonCommercial-NoDerivs 4.0 International License (CC BY-NC-ND 4.0), which permits the noncommercial replication and distribution of the article with the strict proviso that no changes or edits are made and the original work is properly cited (including links to both the formal publication through the relevant DOI and the license). 
See: https://creativecommons.org/licenses/by-nc-nd/4.0/.

\section{References}

1. Ishikawa $\mathrm{K}$, Tilemann L, Ladage $\mathrm{D}$, et al. Cardiac gene therapy in large animals: bridge from bench to bedside. Gene Ther 2012;19:670-7.

2. Yuan QY, Huang J, Chu BC, et al. A targeted highefficiency angiogenesis strategy as therapy for myocardial infarction. Life Sci 2012;90:695-702.

3. Pagano F, Picchio V, Chimenti I, et al. On the Road to Regeneration: "Tools" and "Routes" Towards Efficient Cardiac Cell Therapy for Ischemic Cardiomyopathy. Curr Cardiol Rep 2019;21:133.

4. Shi H, Xue T, Yang Y, et al. Microneedle-mediated gene delivery for the treatment of ischemic myocardial disease. Sci Adv 2020;6:eaaz3621.

5. Neal JM. Ultrasound-Guided Regional Anesthesia and Patient Safety: Update of an Evidence-Based Analysis. Reg Anesth Pain Med 2016;41:195-204.

6. Dietrich CF, Lorentzen T, Sidhu PS, et al. An Introduction to the EFSUMB Guidelines on Interventional Ultrasound (INVUS). Ultraschall Med 2015;36:460-3.

7. Maeda K, Seymour R, Ruel M, et al. EchocardiographyGuided Intramyocardial Injection Method in a Murine Model. Methods Mol Biol 2017;1553:217-25.

8. Giraldo A, Talavera López J, Fernandez-Del-Palacio MJ, et al. Percutaneous Contrast Echocardiography-guided Intramyocardial Injection and Cell Delivery in a Large Preclinical Model. J Vis Exp 2018;(131):56699.

9. Prendiville TW, Ma Q, Lin Z, et al. Ultrasound-guided transthoracic intramyocardial injection in mice. J Vis Exp 2014;(90):e51566.

10. Liu L, Li J, Zuo L, et al. Percutaneous Intramyocardial Septal Radiofrequency Ablation for Hypertrophic Obstructive Cardiomyopathy. J Am Coll Cardiol 2018;72:1898-909.

11. Zhou Q, Deng Q, Hu B, et al. Ultrasound combined with targeted cationic microbubble-mediated angiogenesis gene transfection improves ischemic heart function. Exp Ther Med 2017;13:2293-303.

12. Ling ZY, Shu SY, Zhong SG, et al. Ultrasound targeted microbubble destruction promotes angiogenesis and heart function by inducing myocardial microenvironment change. Ultrasound Med Biol 2013;39:2001-10.

13. Sun Z, Xie Y, Lee RJ, et al. Myocardium-targeted transplantation of PHD2 shRNA-modified bone mesenchymal stem cells through ultrasound-targeted microbubble destruction protects the heart from acute myocardial infarction. Theranostics 2020;10:4967-82.

14. Wang Z, Jiang S, Li S, et al. Targeted galectin-7 inhibition with ultrasound microbubble targeted gene therapy as a sole therapy to prevent acute rejection following heart transplantation in a Rodent model. Biomaterials 2020;263:120366.

15. Yang L, Yan F, Ma J, et al. Ultrasound-Targeted Microbubble Destruction-Mediated Co-Delivery of Cxcl12 (Sdf-1alpha) and Bmp2 Genes for Myocardial Repair. J Biomed Nanotechnol 2019;15:1299-312.

16. Cao S, Zhou Q, Chen JL, et al. Comparison of Intracoronary and Intravenous Ultrasound-targeted Microbubble Destruction-mediated Ang1 Gene Transfection on Left Ventricular Remodeling in Canines With Acute Myocardial Infarction. J Cardiovasc Pharmacol 2017;70:25-33.

17. Cao S, Deng Q, Wang Y, et al. Ultrasound-targeted microbubble destruction-mediated Ang1 gene transfection improves left ventricular structural and sympathetic nerve remodeling in canines with myocardial infarction. Ann Transl Med 2021;9:221.

18. Shohet RV, Chen S, Zhou YT, et al. Echocardiographic destruction of albumin microbubbles directs gene delivery to the myocardium. Circulation 2000;101:2554-6.

19. Deng Q, Hu B, Cao S, et al. Improving the efficacy of therapeutic angiogenesis by UTMD-mediated Ang-1 gene delivery to the infarcted myocardium. Int J Mol Med 2015;36:335-44.

20. Bonnet G, Ishikawa K, Hajjar RJ, et al. Direct Myocardial Injection of Vectors. Methods Mol Biol 2017;1521:237-48.

21. Scimia MC, Gumpert AM, Koch WJ. Cardiovascular gene therapy for myocardial infarction. Expert Opin Biol Ther 2014;14:183-95.

22. Lavu M, Gundewar S, Lefer DJ. Gene therapy for ischemic heart disease. J Mol Cell Cardiol 2011;50:742-50.

23. Hinkel R, Trenkwalder T, Kupatt C. Gene therapy for ischemic heart disease. Expert Opin Biol Ther 2011;11:723-37.

24. Kim J, Mirando AC, Popel AS, et al. Gene delivery nanoparticles to modulate angiogenesis. Adv Drug Deliv Rev 2017;119:20-43.

25. Fujii H, Sun Z, Li SH, et al. Ultrasound-targeted gene delivery induces angiogenesis after a myocardial infarction in mice. JACC Cardiovasc Imaging 2009;2:869-79.

26. Sun L, Cui M, Wang Z, et al. Mesenchymal stem cells modified with angiopoietin-1 improve remodeling in a rat model of acute myocardial infarction. Biochem Biophys 
Res Commun 2007;357:779-84.

27. Rufaihah AJ, Johari NA, Vaibavi SR, et al. Dual delivery of VEGF and ANG-1 in ischemic hearts using an injectable hydrogel. Acta Biomater 2017;48:58-67.

28. Li SH, Lai TY, Sun Z, et al. Tracking cardiac engraftment and distribution of implanted bone marrow cells:

Comparing intra-aortic, intravenous, and intramyocardial delivery. J Thorac Cardiovasc Surg 2009;137:1225-33.e1.

29. Thiagarajan H, Thiyagamoorthy U, Shanmugham I, et al. Angiogenic growth factors in myocardial infarction: a

Cite this article as: Cao S, Deng Q, Tan T, Zhou Y, Wang Y, Zhou Q. Transthoracic ultrasound-guided percutaneous intramyocardial injection combined with ultrasound-targeted microbubble destruction-mediated angiogenin 1 gene therapy in canine myocardial infarction model. Cardiovasc Diagn Ther 2021;11(6):1190-1205. doi: 10.21037/cdt-21-364 critical appraisal. Heart Fail Rev 2017;22:665-83.

30. Lu ZY, Li RL, Zhou HS, et al. Rescue of hypertensionrelated impairment of angiogenesis by therapeutic ultrasound. Am J Transl Res 2016;8:3087-96.

31. Baldazzi F, Jørgensen E, Ripa RS, et al. Release of biomarkers of myocardial damage after direct intramyocardial injection of genes and stem cells via the percutaneous transluminal route. Eur Heart J 2008;29:1819-26. 